\title{
EL ROL DE LA MUJER CHINA A PRINCIPIOS DEL SIGLO XX EN LA NOVELA BANSHENG YUAN DE ZHANG AILING
}

\author{
THE ROLE OF CHINESE WOMAN IN EARLY TWENTIETH CENTURY IN ZHANG \\ AILING'S NOVEL BANSHENG YUAN
}

Teresa Inés Tejeda Martín

Universidad de Salamanca

\section{RESUMEN:}

En este artículo reflexionaremos, a través de la lectura Bansheng yuan, la primera novela larga de Zhang Ailing (Eileen Chang), sobre la situación de la mujer china en las primeras décadas del siglo XX. Con el análisis de los tres principales personajes femeninos nos centraremos en destacar dos aspectos que, a pesar de haber avanzado en la teoría, seguían lastrando la posición de la mujer en la práctica: la falta de independencia laboral y el matrimonio.

\section{Palabras clave:}

Zhang Ailing, Bansheng yuan, emancipación de la mujer, mujer china

\section{Abstract:}

In this paper, we will reflect on the condition of Chinese women in the first decades of the 20th century through a close reading of Bansheng yuan, Zhang Ailing (Eileen Chang)'s first long novel. Through the analysis of the three main female characters, we will focus in two aspects -lack of labor independence and marriage- to underscore that, in spite of some theoretical improvements, the position of women was still burdened in reality.

\section{KeYWORDS:}

Zhang Ailing, Bansheng yuan, women emancipation, Chinese women 
Las primeras décadas del siglo XX en China son testigos de fascinantes cambios, especialmente para la mujer, que tras años de lucha conquistará muchos derechos que hasta el momento le habían sido negados. En la literatura encontramos voces femeninas que reivindican cada vez con más fuerza un lugar propio en la nueva sociedad. Zhang Ailing, una escritora con gran éxito en el Shanghai de los años cuarenta, no se alza entre las escritoras que hacen gala de un discurso revolucionario, sin embargo, sus escritos tienen el potencial de reflejar, a través del comportamiento, en las palabras y en el silencio de sus personajes, los conflictos a los que se enfrentaba una mujer burguesa del momento. Por ello, se propone aquí la lectura de una de sus novelas, Bansheng yuan 半生缘 ${ }^{1}$, que ya en su concepción destila características de una época convulsa. Esta obra clave nos permite reflexionar en torno a dos pilares que determinaban el rol que desempeñaba la mujer en su sociedad: su posición en el mundo laboral y el matrimonio.

\section{LA MUJER EN CONFLICTO: ENTRE LA ANTIGUA Y LA NUEVA SOCIEDAD}

La mujer ha ocupado un rol subalterno dentro de la sociedad y la cultura china tradicional, por lo que antes de abordar la obra de Zhang Ailing se hace necesario atender a los cambios sucedidos entre el final de la dinastía Qing (1911), la República China (1912-1949) y la fundación de la República Popular China (1945). Estos permitieron que la mujer saliera de los cuartos interiores a los que había sido largamente relegada y comenzara a participar activa y públicamente en la sociedad. Al dibujar este contexto pretendemos subrayar que, a pesar de encontrarnos con transformaciones que revolucionaron la vida de la mujer, el gran peso de la tradición seguía restringiendo en gran medida su verdadera emancipación, por las limitaciones fundamentalmente ideológicas que se le imponían en el mundo laboral, como las que conllevaba, en muchas ocasiones, el matrimonio. Profundizaremos en estos dos puntos en los siguientes párrafos.

Hemos de aclarar también que nos centraremos exclusivamente en la mujer burguesa por dos motivos principales: en primer lugar, porque fue quien lideró y a quien se dirigieron los primeros movimientos feministas, $y$, en segundo lugar, porque es el ámbito al que pertenece tanto Zhang Ailing como los personajes de Bansheng yuan a los que nos referiremos. Esbozaremos en las siguientes páginas el nacimiento del feminismo en China.

1.1. Posición de LA MUJer en la china imperial

Entre finales del siglo XIX y principios del XX se vivieron en China grandes acontecimientos que no solo marcaron el imparable declive y definitiva caída de un sistema dinástico que había pervivido durante más de dos mil años, sino que también 
marcaron el camino hacia la liberación de la mujer. Hasta el siglo XX la posición de inferioridad que la mujer ocupaba frente al hombre había sido impuesta por un sistema patriarcal que se apoyaba en un orden jerárquico basado en criterios de edad y género, y que había sido establecido principalmente por el confucianismo, sistema de pensamiento predominante. La dicotomía sobre la que se construyó tradicionalmente el concepto de género provenía del principio del yin y yang, en el que se asociaba a la mujer con lo inferior, lo pasional, o la oscuridad, y al hombre con los valores positivos contrarios. Esto implicaba que la mujer debía vivir sometida al hombre, ya fuera como esposa, hija o madre. ${ }^{2}$ Como consecuencia de este sistema social segregacionista se crearon dos esferas diferenciadas: el hombre ocupaba el exterior, es decir, el ámbito público y la mujer veía su vida recluida a los cuartos interiores (Raphals 1998: 1-2).

Ante este contexto general, se entendía que el principal propósito de la mujer consistía en ser una buena madre y una buena esposa, por lo que no es de extrañar que su educación se entendiera como un medio para conseguir ese fin. En las familias de clase alta se solía enseñar a las niñas a leer y escribir con el objetivo primordial de que pudieran hacerse cargo de los asuntos de la casa del futuro marido y educar a sus hijos. Esto, en la mayoría de los casos, limitaba la propia formación que recibía la mujer, ya que al asumir que no participaría en asuntos públicos, su instrucción se centraba a menudo en un corpus de libros elaborados a lo largo del período imperial y diseñados para hacerla conocer sus deberes como hija, nuera, madre, esposa y viuda, y modelar su comportamiento moral (Idema y Grant 2004: 12). Por ello, y a pesar de que a finales de la dinastía Qing se había alcanzado un gran alto grado de alfabetización entre las mujeres, su educación se observaba como un valor para concertar un matrimonio más que como una manera de que consiguiera independencia o incluso jugara un papel social más allá del ámbito privado ${ }^{3}$.

Aunque sería un error afirmar que la mujer ha estado completamente al margen de la esfera pública, pues, por ejemplo, la historia literaria china cuenta con un buen número de escritoras que consiguieron conservar, publicar su obra o alcanzar cierta relevancia. Aun así, la realidad es que durante toda la China imperial la escritura era fundamentalmente un trabajo de hombres, en tanto que la mujer quedaba excluida del dominio oficial y/o profesional. ${ }^{4}$ Hasta el siglo XX la obra de la mujer va a ser

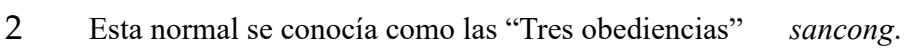

3 Ya a finales del siglo XVIII se produjo un debate sobre lo que las mujeres deberían aprender cuando la obra de muchas escritoras adquirió visibilidad en el panorama literario. En este sentido es relevante la postura de Zhang Xuecheng, quien sin cuestionar que las mujeres debían ser educadas, algo que parecía aceptado ya en su época, sí que hacía énfasis en la necesidad de mantener la separación de las esferas y, por tanto, criticaba directamente la participación de la mujer en el ámbito público. (Mann, 1992: 44)

4 Huelga decir que la situación de la mujer ha variado a lo largo de la historia, y que la historia China cuenta con un considerable número de mujeres que obtuvieron cierta influencia y relevancia tanto en el gobierno como en el campo de las letras, sin embargo, suponían generalmente una excepción. Especialmente desde el giro conservacionista del neoconfucianismo en el siglo XVI. 
concebida como una escritura marginal y, a ojos del hombre y de la mayoría de las mujeres, el que una mujer de clase alta escribiera, si bien era posible, seguía siendo un escándalo (Idema y Grant 2004: 4). ${ }^{5}$

El salto de la mujer al mundo profesional se produce junto con los grandes cambios históricos que dan nacimiento a la China moderna, y los movimientos de liberación de la mujer que los acompañan. Uno de los primeros será la campaña antivendaje de pies que, a finales del siglo XIX, abrirá este largo camino hacia la autonomía femenina ${ }^{6}$. La costumbre del vendado de pies bien sea abordada desde su dimensión erótica, o bien como símbolo último de sumisión, cosificaba y restringía drásticamente la movilidad de la mujer. Pero esta y otras restricciones impuestas durante siglos, en mayor o menor medida, comenzaron a superarse en un proceso que discurre paralelo con el cambio de régimen. Y es que los problemas internos, en especial la Rebelión de los Taiping (18511864), y diferentes conflictos armados con potencias extranjeras agravados por las crisis nacionales (como fueron las Guerras del Opio y la Primera Guerra sino-japonesa) propiciaron la petición generalizada de reformas al gobierno chino, así como también facilitaron la entrada de corrientes de pensamiento extranjeras, principalmente tras la obligada concesión de territorios ${ }^{7}$.

Para Zheng Jiaran la Rebelión de los Taiping podría considerarse como la primera vez que se reclama la igualdad entre hombres y mujeres, sin embargo, esto no acabó conformando el primer feminismo chino ya que este discurso quedaba supeditado a las necesidades de la guerra (2016: 2). En efecto, encontramos en esta revolución campesina grandes líderes militares mujeres y, entre las reformas exigidas, la petición de abolición del vendaje de pies. Pero no solo debemos mencionar la falta de recorrido de estas primeras ideas feministas por su propósito inicialmente práctico, sino, y de acuerdo con Fan Hong, más bien lo debemos achacar a una sociedad aún muy apegada a una tradición confuciana que se resistía a asumir ciertos cambios (2005: 30). Será algunas décadas más adelante cuando asistamos a la consolidación del feminismo en China.

5 Es importante subrayar que durante siglos la mayor parte de literatura escrita por mujeres que circulaba públicamente era la de las cortesanas, especialmente durante la dinastía Tang. Véase Ping Yao: "The Status of Pleasure: Courtesan and Literati Connections in T’ang China (618-907)”. Será a partir de la dinastía Ming cuando las mujeres de familias de elite sean las que poco a poco reemplacen a las cortesanas como principales escritoras. Sin embargo, siempre se consideraba una ocupación secundaria, algo ocioso, y no como una profesión.

6 La dinastía Qing curiosamente supone el cénit de esta práctica, así como su declive. Véase: Wang Ping, Aching for Beauty.

$7 \quad$ La finalización de la Primera Guerra del Opio entre Inglaterra y China con la firma del Tratado de Nanking en 1842 por el que China cedía la isla de Hong Kong y los puertos de ciertas ciudades como Xiamen, Fuzhou, Ningbo, Shanghai y Guangzhou estarían abiertos al comercio internacional. China, que había permanecido cerrada a cierto tipo de comercios se vio forzada a doblegarse ante un país extranjero, lo cual supuso como el principio del fin del imperio, así como una serie de humillaciones para el país. Y Japón: Tratado de Shimonoseki (1895) abre un nuevo período en la historia del mundo chino, su alienación. Por este tratado cede el control de Taiwán, las Islas de los Pescadores y lo que es hoy la provincia de Liaoning. (Fan Hong, 2005: 26) 
La llegada de potencias extranjeras a China propició varios cambios en el panorama nacional. Entre ellos, permitió la entrada de misioneros y con estos la fundación de las primeras escuelas para niñas ${ }^{8}$. Hasta el momento la educación para mujeres se había desarrollado en el ámbito privado del hogar, con tutores, pero gracias a estos primeros centros las mujeres educadas consiguieron llamar la atención de sus compatriotas e ir desterrando la idea de que las mujeres debían permanecer en sus casas. Pronto, algunos reformistas como Kang Youwei (1858-1927), Liang Qichao (1873-1929), Tan Sitong (1865-1898) empezaron a pedir que se abrieran escuelas chinas de mujeres; la primera se funda en 1898 en Shanghai, y se populariza a partir de 1907, momento en el que el gobierno abre muchas más por todo el país (Fan Hong 2005: 60). También en este momento, se comienza a erradicar la tradición de vendar los pies entre las familias más educadas y progresistas (65). No debemos olvidar que junto a estas figuras surgen voces que, aún teniendo menos impacto en el panorama nacional, tuvieron gran relevancia para la mujer, como las de las escritoras Qiu Jin (1975-1907) y He-Yin Zhen (1884-1920?) $)^{9}$ que comienzan a reclamar igualdad e independencia para la mujer.

A principios del siglo $X X$ muchas mujeres, en su gran mayoría de familias acomodadas y que ansiaban ese cambio social aprovecharon la oportunidad que se les brindaba para abandonaron sus "cuartos interiores" y convertirse en escritoras, viajeras o periodistas, y comenzaron a fundar sociedades, academias, revistas, etc. (Yan Haiping 2006: 5). Sin embargo, su participación política no sólo siguió estando limitada sino prohibida, ya que a pesar de que muchas mujeres, como Qiu Jin, habían participado de forma directa en los movimientos revolucionarios que condujeron a la instauración de la República China en 1911, todas ellas vieron cómo tras su fundación se les prohibió formar parte del gobierno, devolviéndoles de nuevo a segundo plano ${ }^{10}$. Esto supuso una gran decepción, ya que la mayor parte de feministas de finales de la dinastía Qing (una de las excepciones sería He Yin-Zhen) estaban íntimamente relacionadas con los movimientos antidinásticos (Hershatter 2007: 84).

Y así nos lo recuerda Amy Dooling al afirmar que este primer feminismo rápidamente fue absorbido por la necesidad más imperante de conformar un nuevo estado y, más importante aún, que los gestos de muchos reformistas tanto de finales del siglo XIX como de las décadas posteriores se apoyaban en un persistente paternalismo

8 La primera se fundó en 1844 en Ningbo (Sáiz, 2001: 51). Para 1867 ya había más de 121 escuelas repartidas por los principales territorios, aunque obviamente no llegaron a todas las regiones (Fang Hong, 2005: 53).

9 También conocida como He Zhen, y definida como escritora anarco-feminista. "Yin" es su apellido materno que la escritora decidió unir al paterno (Liu, Karl, y Ko, 2013: 2), lo que podemos entender como una declaración de intenciones.

10 Incluso se eliminó la gimnasia que había formado parte del currículo de las escuelas de mujeres, ya que se asociaba actividades revolucionarias (Fan Hong, 2005: 102). 
que plantea alguna duda sobre si realmente defendían la completa emancipación femenina, ya que la mujer durante las grandes reformas siguió ocupando una posición marginal y era poco más que una beneficiaria pasiva de la autoridad masculina (2005: 2). Siguiendo este pensamiento, en estas páginas se pretende demostrar con el análisis de la novela de Zhang Ailing, que sin cuestionar las oportunidades y los derechos legales que estas reformas supusieron, sí que debemos interrogarnos sobre el dilema social de la emancipación femenina, no de iure sino de facto.

Tras un período de retroceso y creciente decepción, tras la firma del Tratado de Versalles al finalizar la Primera Guerra Mundial por el que se cedían los derechos coloniales de Alemania en China a Japón, irrumpe en el panorama nacional lo que se ha conocido como el Movimiento del Cuatro de Mayo o el Movimiento por la Nueva Cultura en 1919, liderado por un grupo de jóvenes que apostaban por la renovación, poniendo especial énfasis en la educación. De este modo, se volvieron a poner sobre la mesa demandas como la coeducación, la igualdad y los derechos de la mujer. Las publicaciones escritas y dirigidas hacia mujeres en las que se promovían estas ideas aumentaron, y escritoras como Ding Ling (1904-1986) o Bai Wei (1894-1987) adquirieron cada vez más importancia. También el año 1920 fue significativo ya que se admitieron a las primeras mujeres en la Universidad de Pekín, hecho que fue pronto secundado por otras universidades del país (Yan Haiping 2006: 70).

Durante las primeras décadas del siglo XX hasta la instauración de la República Popular China en 1949 hubo muchos altibajos en la situación de la mujer en la sociedad, que se relacionan en cierta medida con las diferencias y la oposición entre el gobierno del Guomindang y el Partido Comunista Chino, fundado en 1921. Los primeros defendían un papel de la mujer liberada más tradicional, y aunque se reconocían sus derechos a trabajar, su principal misión seguía siendo la familia; mientras, los comunistas eran más radicales y no solo circunscribían su discurso a las familias burguesas y acomodadas, sino que aspiraban incluir en el movimiento a las campesinas y trabajadoras. En lo que atañe a la mujer, estos dos partidos llegaron a cooperar y crearon un organismo llamado Departamento de Mujeres, que desgraciadamente llegó a un abrupto fin en 1927 cuando el presidente Chiang Kai-shek lanzó un ataque contra los comunistas durante lo que se conoció como el Terror Blanco. Yan Haiping llama la atención sobre el hecho de que, si bien el porcentaje de fallecidas fue menor que el de los hombres teniendo en cuenta la proporción en la participación, hubo especial ensañamiento y crueldad en la matanza de mujeres pretendiendo demostrar así su oposición al activismo femenino (2006: 101) ${ }^{11}$. Tras este episodio, algunas comenzaron a reorganizarse en secreto, pero a menudo fueron denunciadas y ejecutadas. El mensaje más contundente

11 Gilmartin recoge en su estudio sobre las mujeres revolucionarias la siguiente aproximación: "Cai Chang estimated in her discussions with Helen Snow that the Nationalist troops killed more than one thousand Communist women organizers and leaders during the first year of the White Terror" (1995: 199). 
se lanzó cuando ejecutaron públicamente a Xiang Jiangyu, que había liderado el sector del Partido Comunista relacionado con los asuntos de la mujer y había codirigido el Departamento de la Mujer hasta 1925, consiguiendo admirablemente compaginar las agendas de ambos lados en este ámbito (Gilmartin 1995: 199).

Inevitablemente esto supuso la revalorización de un modelo de mujer mucho más tradicional en las zonas nacionalistas, pretendiendo así alejarla de la agenda política. Sin embargo, las bases para la independencia de la mujer ya se habían sembrado, por lo que nos encontraremos con una sociedad llena de contradicciones de la que se hará eco la literatura y también el cine. Es precisamente en este contexto disonante y en transformación donde se ubica la obra que aquí analizaremos, Bansheng yuan. En ella se retratan personajes que, sin pretensiones políticas o reivindicativas, intentan avanzar con los tiempos, pero se ven frecuentemente estancados en un rol impuesto por la mentalidad más conservadora con el que cada vez le cuesta más identificarse. En el siguiente epígrafe presentaremos brevemente a la autora y el determinante proceso gestacional de esta obra.

\section{Zhang Ailing y Bansheng yuan}

Zhang Ailing, también conocida como Eileen Chang (1920-1995), se considera actualmente como una de las mejores escritoras chinas del siglo XX, aunque durante muchos años su obra se pasó por alto en la China continental, en esencia, por motivos ideológicos. Comenzó a publicar durante los años 40 en un Shanghai ocupado por los japoneses, donde alcanzó una gran popularidad. Pero su falta de compromiso con los movimientos revolucionarios, así como el hecho de que su marido fuera acusado de colaboracionista con los japoneses y huyera a Japón, la dejaron en una posición muy delicada tras la victoria del Partido Comunista Chino en 1949. Consiguió irse a Hong Kong en 1952, desde donde emigró a Estados Unidos, y allí permaneció hasta su muerte.

La escritora nació en una familia en declive de la antigua aristocracia de la dinastía Qing, en la que, parafraseando a Julia Lovell, se vio constantemente en disputa entre las fuerzas de la tradición y la modernidad, conflicto que plasmará recurrentemente en sus obras (2007, X). En medio de esa batalla entre los valores más conservadores y las oportunidades que brindaba la nueva sociedad se debaten sus personajes, especialmente los femeninos, de Bansheng yuan que analizaremos en el siguiente apartado. Lovell relaciona este constante disyuntiva con la propia naturaleza de sus progenitores, pues describe al padre de Zhang Ailing casi como un cliché de la sociedad más decadente: fumador de opio, con una concubina, y un patriarca violento; mientras que identifica a su madre con la "mujer moderna", una persona educada y con la independencia suficiente como para divorciarse (2007: X). 
No solo podemos reconocer las contradicciones a las que sus personajes dan vida dentro de su ámbito familiar, sino que también debemos considerar que su obra se desarrolló en unos de los momentos más convulsos de la historia china. A pesar de ello, en sus textos se mantuvo en una posición en cierto modo apolítica, lo que Lovell adjudica por un lado, a la intención de evitar la censura impuesta por los japoneses, y por otro, a su escepticismo personal ante la retórica revolucionaria que muchos de sus compañeros habían adoptado (2007: XII). Esto se comprueba en que, por ejemplo, los conflictos armados aparecen en sus obras poco más que como un telón de fondo en los que nunca se profundiza. Lovell menciona a los japoneses, pero esa autocensura vendría, como se explicará posteriormente, también por el triunfo de los comunistas.

La temática de sus textos gira, a primera vista, en torno de las trivialidades de la vida y el amor, por lo que, a menudo, se la ha relacionado con la escuela de los Patos Mandarines y las Mariposas; algo que algunos de sus críticos han lamentado profundamente por considerarlo un menosprecio (Chang 1988: 204). Explica Rey Chow que esta etiqueta se empleó de manera peyorativa para referirse a aquellas novelas populares y de entretenimiento que seguían estilos más tradicionales y se consideraban, de manera muy reduccionista, como simples historias de amor, cuando, en realidad, bajo esta etiqueta se englobaban novelas sociales, de detectives, de fantasía, legendarias y otros muchos tipos (1991: 36). Esta escuela ha quedado eclipsada por el Movimiento del Cuatro de Mayo en las historias literarias, a pesar de haber sido mucho más popular en su día, por su compromiso político, y algo similar le ha pasado a Zhang Ailing en el discurso literario en la China Continental hasta la década de los 90.

Según la opinión de la profesora taiwanesa Sung-sheng Chang ${ }^{12}$, su obra se puede prestar a una crítica superficial esencialmente por dos motivos: primero, porque el género del romance le ha brindado un considerable éxito comercial y, segundo, porque la crítica ortodoxa menosprecia aquella literatura que lidia con los delicados sentimientos de la mujer (1988: 206). Sin embargo, como se intenta demostrar aquí y reivindican sus defensores, precisamente en ese delicado retrato de la mujer atrapada en los valores tradicionales mientras el mundo cambia a su alrededor reside su valor, pues nos ofrece una ventana desde la que asomarnos al sufrimiento de muchas de sus contemporáneas, que, sin ser activistas, pretendían trascender del limitado rol social que se les imponía. Zhang nos ofrece personajes urbanos, no idealizados, incluso a menudo despreciables, que habitaban el Shanghai de principios de siglo, fruto de esas constricciones sociales e ideológicas. En una entrevista la autora expresaba a propósito de Manlu, uno de los personajes femeninos más controvertidos de la novela que nos ocupa:

12 Zhang Ailing ha tenido una gran influencia en las escritoras taiwanesas, como demuestra Sung-sheng Chang (1988). 
Las principales razones [por las que sacrifica a su hermana] tienen su raíz en la sociedad o en la economía. Esto no quiere decir que su comportamiento pueda perdonarse, pero dado que la sociedad antigua reunía las condiciones que generaba personajes como ella, debemos culpar mayormente a ese sistema social irracional. (Ying Du 2006. Traducción propia)

\subsection{BANSHENG YUAN: UN AMOR Y UN LIBRO DE MEDIA VIDA}

No es lugar aquí para comparar minuciosamente las diferentes versiones que existen de esta novela, sin embargo, se considera necesario hacer algunos apuntes sobre su gestación por las implicaciones ideológicas que conlleva. Esta obra, la primera novela larga de la autora, se empezó a publicar de manera serializada el 25 de marzo de 1950 hasta su finalización el 11 de febrero del siguiente año, bajo el título Dieciocho primaveras (十八春 Shiba chun) en el tabloide Yibao ${ }^{13}$. En noviembre de 1951 se publicó una versión revisada y completa del libro firmada por el pseudónimo Liang Jing 梁京 (Ying Du 2006: 204). Zhang Ailing consiguió publicar esta historia de amor en ese momento, en que el Partido Comunista ya había llegado al poder, por tratarse de un período de transición que duraría apenas un par de años más. En las décadas de los años 30 y 40 Shanghai poseía una industria editorial muy fuerte que no desapareció de la noche a la mañana con el cambio de régimen en 1949. Los tabloides suponían un gran porcentaje de las ventas $y$, en un primer momento, se vieron como una vía para educar a los ciudadanos que lo consumían, por lo que el Partido decidió lanzar dos bajo su tutela, Yibao y Dabao, mientras los demás se habían visto obligados a cerrar sus puertas, ya fuera por imposición o porque sus promotores habían huido a Taiwán (Ying Du 2014: 97-98). Las revistas y los suplementos literarios publicados a partir de entonces se centraron en un contenido más propagandístico y se sometieron a un estricto control para asegurar que se ajustaban a los parámetros literarios del Partido impuestos ya en Yan'an en $1942^{14}$. Sin embargo, los tabloides mantuvieron un carácter más abierto, lo que permitió a autoras como Zhang Ailing y otros escritores reconocidos, incluso de la Escuela de los Patos Mandarines y las Mariposas, continuar publicando (Ying Du 2014: 100). De este modo, la escritora logró publicar dos novelas y dos breves ensayos bajo el nombre de Liang Jing.

13 Los tabloides eran publicaciones que cubrían temas como la literatura trivial, entretenimiento, películas, ópera local, deportes, noticias sociales, cotilleo de famosos y cosas del día a día. Este tipo de publicaciones había existido anteriormente, como una especie de noticias fuera de lo oficial, pero habían adquirido gran importancia desde finales de los Qing y durante la época de la República. Con su popularización nacen tabloides específicamente literarios que se identificaban con la clase urbana del momento (Ying Du: 2014: 95).

14 Nos referimos a las Charlas en el Foro de Yan'an sobre Literatura y Arte 在延安文艺座谈会上的讲话 Zai Yan'an wenyi zuotanhui shang de jianghua celebradas en 1942. En ellas se estableció que la literatura tenía que servir para divulgar los valores del Partido y estar dirigida a los soldados, campesinos y obreros. Tras la victoria del Partido Comunista en 1949 estas directrices se extendieron por todo el país. 
Ying $\mathrm{Du}$, en su análisis sobre las diferencias de estas dos primeras versiones, la serializada y la completa, concluye que en la primera hay una clara intención de la escritora por adaptarse al lenguaje del momento y mostrar una actitud positiva con el nuevo régimen, incluyendo referencias, términos comunistas e incluso antiguas pretensiones revolucionarias en boca de los tres jóvenes protagonistas, pero sin darles ninguna profundidad ni verdadera relevancia. Por este motivo, al eliminar parte de estos diálogos, no vemos afectada la trama principal de la novela en la segunda versión (Ying Du 2014; 2006).

Pero esa no fue la última vez que la autora retocó su novela. Ya en Estados Unidos, la revisó de nuevo y la rebautizó como Bansheng yuan, publicándola en Taiwán en 1969. Karen S. Kingsbury (2014), en el prólogo de la traducción de esta obra al inglés, bajo el título Half a Lifelong Romance, apunta que la experiencia acumulada de Zhang Ailing en esos años hará que esta última versión tenga un tono algo más sobrio que las anteriores. Pero no solo cambia el tono, sino que también modifica el final, ya que en el primer texto los personajes se liberan de sus modos burgueses y mejoran sus estándares morales, en un claro guiño a la retórica del momento, y, además, vuelven a reunirse con la pareja de la que estaban verdaderamente enamorados, quizás en un intento de mostrar una sociedad más justa que había roto con la anterior. En opinión de Kingsbury (2014), no es sorprendente que en su última versión la escritora alterara estos eventos y eliminara cualquier referencia directa a comunistas o nacionales y se centrara únicamente en el devenir de los personajes alejándose de cualquier compromiso forzado con un bando. Y es que, como mencionamos más arriba, el discurso político resulta ajeno a su obra y, hasta se podría decir que extraño a la realidad que quiere reflejar. Con esta premisa reduce los dieciocho años iniciales de la trama a catorce que van de 1931-1945, eliminando los últimos años de guerra civil entre los dos bandos chinos. ${ }^{15}$

En este estudio tomaremos como referencia la última versión, ya que consideramos que no solo se ajusta más a las particularidades de sus trabajos, sino que no se ve contaminada por un discurso revolucionario idealizado e impuesto, y refleja de manera más independiente las contradicciones y conflictos de la posición de la mujer en la época. En nuestro análisis queremos trascender la imagen que, en ocasiones, se ha proyectado de esta novela como una historia de amor o una novela rosa ${ }^{16} \mathrm{y}$ ahondar en lo que aflora en los diálogos y los silencios de sus protagonistas.

15 La única referencia temporal del libro es la Batalla de Shanghai en 1937, tres meses de lucha entre los ejércitos chinos y japonés, en el capítulo 15, en ese momento Gu Mazhen tiene unos 30 años (Zhang Ailing tenía 17 en ese momento). Sus biógrafos indican que ella escapó de cualquier daño relacionado con la guerra, aunque en ese período sufrió de violencia doméstica y su padre la encerró (como le sucederá a su personaje protagonista) hasta que consiguió escapar al apartamento de su madre con la ayuda de una criada.

16 Tras la muerte de la autora, esta historia ha sido adaptada al cine, televisión e incluso a musical, tanto en Hong Kong, China como Taiwán, si bien cada productor ha seguido una versión diferente para hacerlo. Lo que comparten, en general, es la imagen que proyectan principalmente como historia de amor. 


\section{TRES MUJERES DE LA FAMILIA GU: ENTRE LA DEPENDENCIA Y EL SACRIFICIO}

El argumento de la novela se podría simplificar como una historia de amor truncada entre cuatro jóvenes. La trama comienza recordando cómo se conocieron los dos principales protagonistas: Manzhen y Shijun catorce años atrás, cuando trabajaban en una fábrica. Entonces, eran un grupo de tres, al que se añadía Shuhui, compañero y amigo de Shijun, que se enamora de Cuizhi, una amiga de la infancia de Shijun. Sin embargo, serán Shijun y Cuizhi quienes se casen por despecho, mientras que Shuhui y Manzhen acabarán divorciados tras un matrimonio fallido y otro obligado respectivamente. De esta forma, de las dos posibles parejas ideales o predestinadas, todos terminan o con la persona equivocada o solos. Son varios los personajes que merecerían comentario, como la joven Cuizhi, de familia de clase alta a quien no se le permite cursar estudios superiores y se la presiona para que se case cuanto antes, pero este análisis se centrará en tres de los personajes femeninos de la misma familia: las hermanas Gu Manzhen, $\mathrm{Gu}$ Manlu, y su madre, la señora $\mathrm{Gu}$, y en cómo se posicionan dentro de los valores éticos, morales y sociales que condicionaban la vida de la mujer de su época. A través de ellas se reflexionará sobre los principales conflictos ocasionados por la pervivencia de un sistema que seguía constriñendo el lugar que ocupaba la mujer en el mundo, tanto el de las que seguían atrapadas en las maneras más tradicionales de entender su papel, como en el de las que intentaban romper con las restricciones impuestas por la anterior generación.

3.1. LA INDEPENDENCIA Y EL ACCESO AL MUNDO LABORAL

En la trama, la situación familiar de estas tres mujeres se vio alterada de manera definitiva al morir el cabeza de familia, lo que nos remite inmediatamente al modelo de familia tradicional. El padre de Manzhen y Manlu trabajaba en una librería y con un sueldo muy humilde mantenía a su mujer, su madre y cinco hijos. Al fallecer, la familia se quedó sin sustento y la hermana mayor, Manlu, que no había acabado aún los estudios de secundaria, tuvo que sacrificarse para mantener a toda la familia, romper su compromiso con un joven del que estaba enamorada y convertirse en lo que podríamos traducir como una acompañante de baile "de alquiler" en los salones. ${ }^{17}$ En principio, este trabajo no llevaba implícita una actividad relacionada con la prostitución, pero, como explica Manzhen a Shijun: "Claro que hay chicas de baile que no se corrompen, pero esas no pueden mantener a una familia grande" (Zhang Ailing

17 En chino 舞女 wunü, literalmente “chica de baile” y en inglés “taxi-dancer”. 
El rol de la mujer china a principios del siglo XX en la novela Bansheng yuan de Zhang Ailing 
2012, Cap. 2). ${ }^{18}$ Manzhen se lamenta de que su hermana tenga que seguir ese camino porque es consciente de que no hay vuelta atrás, y reivindica su buen corazón, ya que gracias a su voluntad ella sí ha podido estudiar y encontrar un trabajo como secretaria en una fábrica.

El que sea la hermana mayor y no la madre la que tome el relevo de su padre, ya nos muestra una imagen de la señora $\mathrm{Gu}$ como una mujer producto de la sociedad antigua, completamente dependiente de los demás y sin recursos para valerse por sí misma fuera de su casa. Su personaje análogo será la madre de Shijun, una mujer que vive resignada a que su marido conviva con su concubina y que ni siquiera sabe leer. Manlu ya pertenece a una generación diferente, pero su educación se ve interrumpida por la muerte de su padre y, por tanto, abocada al mismo destino que otras muchas mujeres de la época.

El que una mujer acabara prostituyéndose para poder mantener a su familia, o incluso a ella misma, por falta de otros recursos es algo que ya había preocupado a varios pensadores del momento, que incidían, como Qiu Jin, en lo importante que es para una mujer la educación y la profesionalización. En un sentido similar, Lu Xun (1881-1936), considerado el padre de la literatura moderna china y máximo representante del Movimiento del Cuatro de Mayo, dio una charla en la Universidad Normal para Mujeres de Beijing el 26 de diciembre de 1923, que después publicaría en un artículo titulado "¿Qué sucede después de que Nora abandone su casa?". ${ }^{19}$ y en la que exponía cómo podría acabar una "Nora china" si se fuera de casa, dado que la mujer no estaba realmente emancipada y/o tenía recursos para poder mantenerse a sí misma. Según este autor, las posibilidades más factibles que se le presentaban a una mujer en una situación así eran que cayera en la prostitución, se suicidara, o no le quedase más remedio que volver a su marido. ${ }^{20} \mathrm{Lu}$ Xun advertía, en este sentido, que los sueños estaban muy bien, pero el dinero era esencial: "El dinero no puede comprar la libertad, pero la libertad puede ser vendida por dinero" (Lu Xun, 1980: 88). Con este mensaje, muy similar al que unos años después popularizara Virginia Woolf, pretendía despertar a las mujeres para que fueran conscientes de que tenían que luchar por cambiar sus circunstancias, cosa que no funcionaría si no conseguían ser independientes económicamente. También incidía en que, aunque algunas mujeres habían conseguido hacer valer sus derechos, esa realidad aún no era la norma y quedaba

18 Al tratarse de una versión electrónica del libro no podemos determinar la página, por lo que señalamos el capítulo para que el lector pueda encontrar más fácilmente la referencia. Además, tanto esta como las siguientes citas de la novela serán traducción del chino al español de la autora de este artículo.

19 En 1918 se tradujo al chino la obra de Ibsen La casa de las muñecas, en el que el personaje femenino Nora representaba a la mujer liberada, capaz de enfrentarse a su familia y a la sociedad.

20 En algunas películas de los años treinta asociadas con el Cine del Movimiento de Izquierdas, como son Nueva Mujer o la Diosa reflejan este constante conflicto de una mujer que intenta abrirse paso en la sociedad, pero que ve cómo constantemente se la intenta frenar y acaban o prostituyéndose o siendo víctimas de acosos y difamaciones por resistirse. 
mucho camino por andar. Manlu no huye de casa, pero sí que se ve obligada a venderse a sí misma, precisamente por la incapacidad de su madre y sus propias limitaciones dentro del mundo laboral. Así nos recuerda también Zhang Ailing, que, si bien la mujer no tenía prohibida su actividad laboral, y por tanto su emancipación económica, sus posibilidades quedaban realmente muy reducidas por su pobre educación.

Pese a esta situación, y aunque toda la familia vive de Manlu y de sus amantes, en la novela se marca una separación moral entre ellos, que se manifiesta físicamente dentro de la vivienda: Manlu y su sirvienta ocupan el piso inferior y el resto de la familia habita en el segundo, "un mundo completamente diferente" (Zhang Ailing 2012, Cap. 2). La madre y la misma Manlu, son especialmente protectoras en un primer momento con Manzhen, ya que representa las oportunidades que a ellas les han sido negadas: una educación completa, independencia económica y pureza moral. Esto provoca un sentimiento de extrañeza entre las dos hermanas, como si pertenecieran a mundos diferentes. En una escena en la que Manzhen pasa al lado de su hermana por la escalera, la observa de la siguiente manera:

[Manlu] Llevaba puesto un qipao largo de satén suave verde manzana, que, a pesar de ser prácticamente nuevo tenía una leve marca negra de una mano a la altura de la cintura, dejada por el sudor de la gente con la que bailaba. La aparición repentina de una huella renegrida en la ropa tenía algo de terrorífico. Su pelo estaba revuelto, todavía sin peinar, sin embargo, ya llevaba su maquillaje de la pista de baile, rojo escarlata y negro azabache, con sombra azul alrededor de los ojos. De lejos había que admitir que estaba guapa, pero de cerca tenía una apariencia más bien siniestra. Manzhen se apartó al subir por la escalera, como distraída, no podía creerse que esta fuera su hermana mayor. (Zhang Ailing 2012, Cap. 2. Traducción propia)

Y es que realmente ya no formaban parte de la misma esfera, ya que cuando Manlu se casa con uno de sus amantes y se va de casa, Manzhen toma las riendas de la familia y, compaginando varios trabajos y alquilando el piso inferior de la vivienda, resulta capaz de mantener a toda la familia de una manera que la sociedad consideraba moralmente aceptable. Pero no podemos pasar por alto el hecho de que, a pesar de que fueran dos mujeres las que consiguieran llevar toda la carga familiar sobre sus hombros, al final esto no las otorgaba más independencia, dado que continuaban siendo esclavas de los valores de su sociedad. Y es que como mencionamos en el primer apartado, la principal ocupación de una mujer de la época seguía siendo su papel como madre y esposa, y así se comprobará en el destino de ambas hermanas.

\subsection{LA INDEPENDENCIA Y EL MATRIMONIO: LA MUJER COMO ESPOSA Y MADRE}

Cuando Manlu deja de ser tan joven a ojos de la sociedad, la madre consciente de que ese trabajo no es algo que podrá hacer siempre, la insta para que encuentre 
marido lo antes posible. Aquí comprobamos que las opciones de este personaje ya "desgastado" se reducen de nuevo a la dependencia de un hombre, permaneciendo irremediablemente atrapada en el inevitable sistema patriarcal. Así pues, cuando Zhu Hongcai, personaje que se describe con repulsión desde el comienzo y que está casado y tiene hijos que residen en una zona rural, se muestra dispuesto a casarse con ella, la madre y la abuela, representantes de la antigua sociedad, lo celebran con gran entusiasmo. En cuanto al matrimonio, debemos aclarar en este punto ciertas peculiaridades del momento.

Tradicionalmente en China un hombre podía casarse con una mujer y tener varias concubinas, que ocupaban un estatus algo inferior que la mujer principal. El Guomindang, partido nacionalista que gobernaba el país, en teoría promovía un matrimonio moderno formado exclusivamente por un hombre y una mujer, y por ello prohibió la bigamia. Sin embargo, eran muchos los interesados en mantener esta tradición, y la ley, en cierto modo, facilitaba que así fuera, ya que el concubinato no se consideraba un "matrimonio" a efectos legales, y se reflejaba como una especie de "acuerdo doméstico". Por ello, es frecuente seguir encontrando en la literatura referencias a un sistema que, a todas luces, se había quedado obsoleto, a la vez que seguía poniendo de manifiesto la desigualdad entre sexos. Esta costumbre se veía avalada legalmente también porque solo se consideraba delito la infidelidad si la cometía una mujer, por lo que el concubinato en última instancia no era ni bigamia ni adulterio. No fue hasta 1931 cuando se reconoció el derecho de la mujer a divorciarse de un hombre si le era infiel, y en 1935 cuando se estableció como una ofensa castigable para ambos sexos. Pero los cambios sociales llegaron más tarde que la ley, y los matrimonios monógamos no se impusieron hasta 1949 (Lynn Pan, 2015: 176-177). La pervivencia de esta costumbre alegal pero socialmente aceptada se refleja en el acuerdo de Manlu con Zhu Hongcai y en su discusión sobre la celebración de la boda, pues el hombre se decanta por una ceremonia discreta precisamente para no "quebrantar" la ley:

“¡Ya sabes que la bigamia es un crimen!” Manlu girando la cabeza le dijo: "La bigamia es un crimen, pero con que tu mujer del campo no diga nada ya está. ¿No dices que a ella le das igual?" Zhu Hongcai contestó: "Ella no se atrevería nunca a hacer algo así, pero tengo miedo a que sea su familia la que diga algo." (Zhang Ailing 2012, Cap. 2)

Esta conversación se ubica a principios de los años 30 y refleja la laxitud social respecto a lo que, a efectos prácticos, era bigamia, a la vez que se manifiesta la pasividad, que entendemos casi como obligada, de la primera esposa. La madre de Shijun, también por absoluta dependencia económica de su marido, se ve forzada a soportar que su marido mantenga y viva prácticamente hasta el final de sus días con su concubina, 
siempre temerosa de no recuperar el favor conyugal y verse desahuciada a su muerte ${ }^{21}$. Al final, Manlu y su prometido celebran abiertamente su boda y se instalan a ojos de la sociedad como marido y mujer en una nueva casa. Desde ese momento, Manlu pasa a estar a disposición de la volatilidad y el capricho de su marido, que cada vez la tratará peor. Lydia H. Liu apunta que la voluntad femenina queda descartada en el discurso tradicional porque la mujer se ve constantemente definida como hija, hermana, esposa y madre (1993: 46), y así lo comprobamos con Manlu y más adelante con Manzhen, pues parece que su lugar en el mundo acaba siempre queda delimitado por la posición que se le concede dentro de la institución familiar.

Manzhen, consciente de las implicaciones del matrimonio, mantiene su compromiso con Shijun en secreto y no quiere casarse inmediatamente con él porque teme que en caso de quedarse embarazada, tenga que dejar de trabajar y la manutención de toda la familia recaiga solo sobre el marido: "Lo he visto un montón de veces, si un hombre además de a su propia familia tiene que mantener a la de su mujer, se ve obligado a conseguir dinero de cualquier manera, hace lo que sea, y, ¿qué pasa entonces con el futuro que tenía?" (Zhang Ailing 2012, Cap. 9). Este argumento no es exclusivo del personaje. En 1927 la escritora Chen Xuezhao (1906-1991) escribía un artículo precisamente en torno al dilema de muchas mujeres con educación que se veían forzadas a volver a su rol de madres y esposas una vez que se habían casado:

When both partners in the so-called "one husband-one wife new family" have careers, the problem of dependency doesn't arise, however, as soon as the woman has a baby, she is no longer able to keep her job and the family's economic burden falls entirely on the man's shoulders. Since there is no public childcare and the average family can't afford a nanny, once the baby is born, the woman has no choice but to look after it herself. (Chen Xuezhao 1998: 171)

Para Chen Xuezhao, esto finalmente provocaba que el hombre volviera a ver a su esposa como a aquellas mujeres analfabetas del pasado, que no eran capaces de ser independientes económicamente y acababan siendo propiedad del hombre: "As a result, these educated, accomplished woman are sacrificed to the hegemony of the self-proclaimed 'new men' of China who still haven't eliminated their own slavish natures" (Chen Xuezhao 1998: 171). Esta escritora sin duda se refiere a aquellos más acordes al pensamiento del Guomindang que, a pesar de apoyar cierta liberación de la mujer, la veían más como un accesorio que apoyara al hombre a construir una nueva nación, y no como una igual. Para Chen Xuezhao esta visión refleja una mentalidad esclavista heredada del confucianismo de la que muchos hombres con ideas progresistas ni siquiera eran conscientes. Debemos recuperar la idea expresada por Amy Dooling

21 En Un amor que destruye ciudades de Zhang Ailing también vemos una actitud muy abierta respecto al concubinato, que incluso lleva a la reprobación familiar hacia una mujer que se divorcia de su marido que tenía dos concubinas. 
sobre el peligro de que fuera el hombre quien formulara la liberación de la mujer, que acabaría conllevando este tipo de problemas. Dos décadas antes que Chen Xuezhao, en 1907, He Yin-Zhen había manifestado ya su preocupación sobre el problema de la voluntad femenina:

The cause of women's rights must be won through women's own efforts. It must not be granted by men. If we allow women's rightful role to be imposed by men, we are renouncing our freedom; and if we allow ourselves to look up to men and ingratiate ourselves to them, whatever rights we obtain in this way are handed to us from above. As we continued to be instrumentalized and remain men's appendages, we would be liberated in name only and our rights could never really be our own. (He Yin-Zhen 2013: 63)

No hay en el discurso de Manzhen un matiz tan revolucionario, ni ve con amargura renunciar a su carrera por la crianza de sus hijos, sino que lo concibe como algo práctico, además de como un impedimento para la carrera de él. Sin embargo, resulta interesante cómo tanto Chen Xuezhao como Zhang Ailing coinciden al manifestar la realidad de la mayor parte de las mujeres educadas una vez que se casaban, fuera cual fuera su aproximación a este conflicto.

Tristemente Manzhen no podrá tomar las decisiones sobre su matrimonio ni su futuro de forma libre. Manlu, conocedora de la atracción que Zhu Hongcai siente por su hermana y en vista de que no es capaz de concebir hijos ${ }^{22}$ por los abortos a los que se sometió en el pasado, decide sacrificar a su hermana para asegurarse el favor de su marido, mostrando quizás de la manera más radical posible hasta qué punto una mujer se podía ver atrapada en el matrimonio por su dependencia económica (recordemos las palabras que citamos de Zhang Ailing al hablar de este personaje). Así, finge estar enferma para que Manzhen pase la noche en su casa, una mansión recién construida en las afueras. En mitad de la noche, el hombre entra en su habitación y la viola, escena que se describe con mucha violencia. Manlu consigue que la madre de ambas se haga cómplice del agravio explicándole que Manzhen quiere denunciarlo a la policía. Entonces, la señora Gu sorprendida dice: “¿Qué? Esta niña no entiende nada, ¿cómo va a dejar que se sepa algo así? Será ella la que caiga en vergüenza" (Zhang Ailing 2012: Cap. 12). Manlu subraya que la vergüenza será extensiva a toda la familia, y que su marido tiene una buena posición, por lo que no se puede permitir que un asunto así circule públicamente. La mujer se las apaña para que su madre deje el asunto en sus manos, otra muestra de la pasividad de este personaje, que en última instancia sacrifica a sus dos hijas por una supuesta moral imposible de sostener desde que alentó a su hija mayor a prostituirse. Ambas estarán de acuerdo en que lo más sensato, ahora, es

22 El que una mujer pudiera ofrecer un hijo varón y por tanto un heredero al marido en la sociedad antigua era fundamental, pero seguimos comprobando hasta tiempos recientes que es un pensamiento que no ha desaparecido por completo. 
que Manzhen se case con su violador, aprovechando que su primera esposa ha muerto y ella podría ocupar ese lugar. Manlu, incluso resalta su buena voluntad, al ceder ese ansiado puesto a su hermana.

Pero en esto también se muestra la diferencia de mentalidad entre las dos generaciones, respecto al tema de la virginidad, pues se describe así el pensamiento de Manzhen tras la violación: "Su idea de la castidad claramente era diferente a las de los tiempos antiguos, no pensaba en lo más mínimo que tuviera que avergonzarse por Shijun" (Zhang Ailing 2012: Cap.12). El dolor que siente el personaje al pensar en su novio se asocia a la pérdida y la incapacidad de comunicarse con él. Es en este punto donde se rompe la historia de amor de Shijun y Manzhen, pero no porque él la rechace, sino porque le hacen creer que ella se ha casado con un antiguo amigo de la familia que la había pretendido, y que la familia al completo se ha mudado a su ciudad de origen. Manzhen permanece secuestrada y aislada en la mansión hasta que da a luz a un niño fruto de la violación. A pesar de la insistencia familiar para que se case con su cuñado, ella se negará en repetidas ocasiones. Después de dar a luz a un niño será cuando recupere la libertad huyendo del hospital, si bien, debe dejar a su hijo recién nacido atrás.

Gracias a sus estudios, Manzhen consigue llevar una vida independiente durante algunos años, trabajando en una escuela y resistiendo la presión familiar. Tanto su madre como su hermana vuelven a buscarla para intentar convencerla de que se case con el hombre que la ha violado y la ha mantenido secuestrada durante meses. En una ocasión su madre le dice: "Sé que no te gusta que te diga que vuelvas. En realidad, tu hermana no tiene mala intención, todo es culpa de Hongcai. Y ya que has tenido al niño, no hay ninguna necesidad de que estés por ahí tú sola pasando penas" (Zhang Ailing 2012 Cap.14). La madre, acorde con el pensamiento antiguo, piensa que es mucho mejor volver a la sombra de un hombre, aunque sea como concubina, que vivir sola. Para la señora Gu su hija es una testaruda e, incluso, una desagradecida con la posibilidad que se le está ofreciendo. En este caso, la "Nora" ha encontrado la manera de sobrevivir en el mundo, pero es la mentalidad conservadora la que intenta arrastrarla de nuevo al hogar.

Y así acabará sucediendo. Tras la muerte de su hermana y al enterarse de que su hijo está enfermo, termina claudicando y casándose con Zhu Hongcai para poder cuidarle. Consciente del error que va a cometer, acaba sacrificándose por su hijo porque la sociedad no le permite estar cerca de él de otra manera. En el último encuentro que tiene con Yujing, un amigo del pasado, este le dice: “"Este tipo de arreglo forzado, es cavarte una tumba y acabar con tu vida» [...] Él pensaba que un matrimonio tenía que ser de mutuo acuerdo, si uno pensaba que iba a sufrir, el otro nunca sería feliz" (Zhang Ailing 2012: Cap. 14). Manzhen está de acuerdo con él, pero se siente acorralada ante 
la imposibilidad de tener a su hijo de otra forma. Se casa y deja de trabajar, quedando atrapada en un matrimonio condenado al fracaso. Aquí recordamos de nuevo a Chen Xuezhao, cuando decía que a priori no había nada malo en que una mujer decidiera dedicarse a ser madre y esposa, excepto que el hombre siguiera ignorando su responsabilidad de ser un buen padre y esposo (1998: 173).

Ese será el caso de Manzhen, ya que su convivencia con Hongcai se convierte en una batalla diaria en la que el hombre ataca a su hijo común para provocarla. Tras varios años de un matrimonio infeliz, comienza a vislumbrar algo de esperanza al descubrir que él tiene una amante y que podría divorciarse de él. Pero es importante la reflexión que la protagonista hace al respecto, pensando en la falta de apoyo de su familia, quienes, aunque nunca les gustó Hongcai no aprobarían su decisión de divorciarse de él:

Parecía que, en su situación, una mujer que ya ha superado los treinta años, solo lo aprobarían en el caso de que su marido la maltratara completamente o que se negara rotundamente a mantenerla, incluso aunque tuviera a otra persona fuera, solo lo considerarían si lo hiciera abiertamente y se lo restregara en la cara. A cualquiera que se lo preguntara pensaría que no tenía razones para divorciarse. (Zhang Ailing 2012, Cap. 15)

Manzhen duda de sus posibilidades de ganar un juicio ya que, a pesar de tener en ese momento la ley de su lado (ya mencionamos que en el 1935 se permitía a la mujer divorciarse de su marido por infidelidad), podría complicarse si él podía invertir más dinero en el juicio que ella. En ese momento Shanghai ya estaba ocupado por los japoneses y no era tan fácil conseguir trabajo: “Tampoco era ya tan joven, ni tenía esa energía, ¿sería capaz de hacerse camino donde no lo había?" (Zhang Ailing 2012, Cap. 15). Manzhen acabará divorciándose de él y viviendo con su hijo, sin embargo, habrá sufrido más de diez años de tormentos, soledad y habrá perdido al amor de su vida.

\section{Conclusiones}

A través de los tres personajes femeninos en los que se ha centrado la lectura hemos visto reflejados algunos de los dilemas a los que se enfrentaban las mujeres educadas pertenecientes a la pequeña burguesía de los años treinta. Una clase privilegiada en su contexto, pero a la vez sujeta a una serie de normas morales y sociales que la condicionaban. En la señora Gu observamos la pervivencia de los valores más conservadores de una sociedad que, a pesar de caminar teóricamente hacia la extinción, seguía determinando la realidad de muchas mujeres. Manlu encarna las limitaciones de muchas mujeres que, a pesar de haber nacido ya en tiempos de la República China, al intentar integrarse en el mundo laboral se veían empujadas hacia la prostitución, por la falta de recursos y, por tanto, de acceso a trabajos bien remunerados. Por su 
parte, Manzhen, libre en principio de esas restricciones, se presenta como una joven educada, con posibilidades de un futuro laboral e incluso de un matrimonio libre, pero ve cómo todos esos proyectos se truncan por las maquinaciones familiares que nacen de un entramado social retrógrado que sigue latente.

Zhang Ailing, perteneciente a la burguesía shanghainesa, nos presenta una historia en la que no encontramos reivindicaciones, pero no por ello es indiferente a los conflictos derivados de una sociedad que seguía vulnerando los derechos de la mujer. En esta obra nos dibuja un mundo en el que la protesta está implícita en el sufrimiento de los personajes, que, si bien nos pueden parecer mezquinos o materialistas, son víctimas de su propio mundo. No se encuentran en este texto personajes heroicos ni revolucionarios, pero sí con retratos que de una realidad compleja y no idealizada. En Manzhen, por ejemplo, se concentran los principales dilemas de la mujer moderna que todavía no había podido ni reivindicar definitivamente su nuevo lugar en el mundo laboral ni definir la nueva manera de concebir un matrimonio. A su vez es la encargada de transmitir cierta esperanza, ya que, a pesar de haberse sacrificado por cumplir el papel de esposa y madre, que aparece repetidamente en la novela como la principal misión de la mujer, consigue romper en cierta medida con ese sol, no sin sufrir por ello. Zhang Ailing nos da la oportunidad en esta novela de reflexionar sobre la condición femenina en unas décadas de grandes transformaciones desde un punto de vista íntimo y no politizado, pero no por eso carente de profundidad.

\section{REFERENCIAS BIBLIOGRÁFICAS}

Chang, E., Half a Lifelong Romance, New York, Random House, 2014.

Chang, S.Y., "Yuan Qiongqiong and the Rage for Eileen Zhang Among Taiwan's "Feminine" Writers", Modern Chinese Literature, v. 4, n. 1/2, Gender, Writing, Feminism, China (1988), pp. 201-223.

Chen, X., "The woes of the Modern Woman", Writing Women in Modern China, New York, Columbia University Press, 1998, pp. 169-173.

Chow, R., Woman and Chinese modernity: the politics of reading between West and East, Minneapolis, University of Minnesota Press, 1991.

Dooling, A., Women's Literary Feminism in Twentieth-Century China, New York, Palgrave Macmillan, 2005.

Fan, H., Footbinding, Feminism and Freedom, London, Routledge, 2005.

Gilmartinn, C.K.., Engendering the Chinese Revolution. Radical Women, Communist Politics, and Mass Movements in the 1920s, Berkeley, University of California Press, 1995.

He, Q., Feminism, Women's Agency, and Communication in Early Twentieth-Century China. The Case of the Huang-Lu Elopement, Illinois, Palgrave Macmillan, 2018. 
Hershatter, G., Women in China's Long Twentieth Century, London, University of California Press, 2007.

Idema, W. y Grant, B., The Red Brush. Writing Women in Imperial China, Cambridge, Harvard University Asia Center, 2004.

Liu, L.H., "Invention and Intervention: The Female Tradition in Modern Chinese Literature" en Gender Politics in Modern China: Writing and Feminism, ed., Tani E. Barlow, Durham and London: Duke University Press, 1993, pp. 33-57.

Liu, L.H., Karl, R.E., y Ko, D., The Birth of Chinese Feminism: Essential Texts in Transnational Theory, New York, Columbia University Press, 2013.

Lovell, J., "Foreword", Lust and Caution, New York, Random House, 2007, pp. I-XIX.

Lu, X., Selected Works of Lu Xun Vol. II, Beijing, Foreign Language Press, 1980.

Mann, S., “Fuxue (Women's Learning) by Zhang Xuecheng (1738-1801): China's First History of Women's Culture.", Late Imperial China, v. 13, n.1 (1992), pp. 40-62.

Pan, L., When true love came to China, Hong Kong, Kong Kong University Press, 2015.

Ping, W., Aching for Beauty: Footbinding in China, Minneapolis, University of Minnesota Press, 2000.

Raphals, L., Sharing the Light. Representations of Women and Virtue in Early China, Albany, State University Press of New York Press. Tusquets, 1998.

Sáiz López, A., Utopía y género: las mujeres chinas en el siglo XX, Barcelona, Bellaterra, 2001.

Yao, P. "The Status of Pleasure: Courtesan and Literati Connections in T'ang China (618-907)." Journal of Women's History, v. 14, n. 2 (2002): 26-53.

Yan, H., Chinese Women Writers and the Feminist Imagination, New York, Routledge, 2006. Yin-Zhen, H., "On the Question of Woman's Liberation", The Birth of Chinese Feminism: Essential Texts in Transnational Theory, New York, Columbia University Press, 2013, pp. 53-71.

Ying, D., "Shanghaiing the Press Gang: The Maoist Regimentation of the Shanghai Popular Publishing Industry in the Early PRC (1949-1956)", Modern Chinese Literature and Culture, v. 6, n.2 (2014), pp. 89-141.

Ying, D. "Cong Shibachun de xiuding kan jiefang chuqi de Zhang Ailing", Zhongguo xiandai wenxue yangjiu conggan, n. 1, (2006), pp. 204-220.

Zhang, A., Bansheng yuan, Beijing Shiyue wenyi chubanshe, 2012 [ebook].

Zheng, J., New Feminism in China. Young Middle-Class Chinese Women in Shanghai, Singapore, Springer, 2016 